Alberta-THY-18/96

hep-ph/9606463

June 1996

\title{
Cosmological Reheating and Self-Interacting Final State Bosons
}

\author{
Rouzbeh Allahverdi and Bruce A. Campbell \\ Department of Physics, University of Alberta \\ Edmonton, Alberta, Canada T6G 2J1
}

\begin{abstract}
We consider inflaton decay to final state bosons with self-interactions of moderate strength. We find that such final state self-interactions qualitatively alter the reheat dynamics. In the case of narrow-band resonance decay, where a quantitative analysis is possible, we show that these final state interactions regulate the decay rate. The phenomenon of parametric amplification is then effectively suppressed, and does not drastically enhance the decay rate and reheat temperature. Detailed applications of our results to realistic classes of inflationary models will be considered elsewhere.
\end{abstract}


Inflationary cosmology [1], provides solutions to the flatness, isotropy and stable relic problems of the standard hot big bang. In these models the universe experiences a period of superluminal expansion during which its energy density is dominated by the potential energy of a scalar field (inflaton). Inflation ends when the inflaton enters the oscillatory regime during which we have a matter-dominated FRW-universe, after which the inflaton decays to relativistic particles (reheating). Reheating represents the crucial transition from the epoch of scalar-field dominated dynamics to a hot FRW universe. After reheating, the universe becomes radiation-dominated and its evolution is just that of the standard hot big bang .

In the standard picture of reheating [1, 2] the effective decay of the inflaton occurs when $\Gamma \simeq H$, where $\Gamma$ is the one particle decay rate, and the reheat temperature of the universe is $T_{R} \sim 0.1(\Gamma)^{\frac{1}{2}}$ (from now on $M_{p l}=1$ and all dimensionful quantities are expressed in these units unless otherwise indicated). In this picture, which we will refer to as the linear regime, perturbation theory is taken to be valid and the occupation number of the final state bosons is assumed to be smaller than one (for fermions this is assured because of Pauli blocking). It has recently been recognized within different approaches [3] - 31 that this picture is incomplete, and nonlinear effects can change it essentially, leading by parametric amplification to an enhanced decay of the inflaton (for a recent review see, e.g. [3]). There are two different possible regimes of such a parametrically amplified decay. In the first the decay occurs over many oscillation times of the inflaton, but experiences parametric amplification with moderately large occupation numbers for the modes of the decay product field; this "narrowband resonance" case is amenable to analytic calculation, and we will be able to analyze the modifications to parametric amplification due to final state-self interactions of the decay products quantitatively. In the second case of "broad-band resonance" the amplification of the decay is so strong that there is explosive decay of the inflaton field on a time-scale not hierarchically longer than the oscillation time, with large occupation numbers for the modes of the decay product field; this scenario is more difficult to analyze quantitatively, but the physical mechanisms which we discuss are of sufficient generality that we expect that they alter the decay dynamics in this case also. 
The consequences of a parametrically amplified decay could be significant, and pose major difficulties in the construction of viable inflationary models. If the decay is very fast, the final state particle modes have, in general, very large occupation numbers and are far from thermal equilibrium; these fluctuations have certain effects similar to very high temperature thermal corrections [9, 12], and after thermalization may themselves lead to high reheat temperatures, with thermal energy densities of order the inflationary energy density. While on the one hand GUT symmetry restoration due to these non-thermal configurations revives GUT scenarios for baryogenesis [23, 16, 17], on the other hand it reintroduces the problem of heavy topological defects whose solution was one of the initial motivations for inflation.

Furthermore, realistic models seeking to implement inflation need to be able to stabilize the required flat potential against radiative corrections. The only presently known method to achieve this in the presence of gauge, scalar potential, Yukawa, and gravitational interactions is by using (approximate) supersymmetry to enforce the appropriate non-renormalization. Parametrically amplified decay of the inflaton, and the resulting efficient reheat, poses a mortal danger to realistic, supersymmetric, inflationary models. In supersymmetric theories the reheat temperature is constrained [32, 33, 34] by the need to avoid thermal overproduction of gravitinos. This leads to an upper bound on the reheat temperature of order $10^{8}-10^{9} \mathrm{GeV}$, which is orders of magnitude below what would be achieved by efficient reheat from amplified inflaton decay. One obvious way to avoid this disaster would be to insure that the inflaton is sufficiently weakly coupled to its bosonic decay products that the decay never experiences parametric amplification. This is typically the case for inflatons in a hidden sector which have only gravitational strength couplings to their (observable sector) decay products. On the other hand, in many models one introduces direct superpotential couplings of the inflaton to the chiral scalars into which it decays, and we wish to consider the nature of the reheat dynamics in such models, and whether they are ruled out by constraints on reheating.

In particular, we will examine the effects that the final state self-interactions of the decay products have on the parametric amplification of the inflaton decay. Because of the large occupation numbers for the modes of the produced decay bosons, we expect that the presence of self-interactions of these bosons will result in large effective masses being induced for these modes. If the bosons are thermalized these may be interpreted as thermal plasma masses 
from self-interaction; more generally they will occur as induced effective plasma mass terms in the mode equations for the decay field. As the mode occupation numbers increase, so do these induced masses, until they equal the mass of the inflaton, cutting off the decay. Decay resumes with the thermalization of the decay products, and their dilution and redshift by cosmic expansion, such that their induced masses dip below the inflaton mass; the system thus proceeds in a quasi-stationary process of decay and dilution such that the induced mass of the decay products is always of order the inflaton mass. This regulates the parametric amplification of the decay, preventing abrupt and efficient reheat. In our discussion we will analyze the effects of final state self-interactions for inflaton decays that would otherwise be in the regime of narrow-band parametric amplification. However, because the dominant effect is a kinematical cutoff of the decay, due to the self-induced plasma mass of the final state decay products arising from their strong self-interaction, we expect similar effects in the case of broad-band resonance. Indeed, recent studies of the broad-band decay regime [7, 10, 14 indicate that the explosive decay from the sequence of higher resonance bands can only effectively produce particles whose mass does not exceed that of the inflaton by more than an order of magnitude. So in this case too there will be a kinematical cutoff due to the final-state self-interaction induced plasma mass of the decay products; although it will now be regulated to be not more than of order ten times the inflaton mass the qualitative effects should be otherwise similar. Applications of our present analysis to realistic classes of supersymmetric inflationary models will be considered elsewhere 31.

As a basis for our subsequent arguments we will consider a chaotic inflation model with the following potential, whose features we take to resemble the generic features of scalar potentials which arise in supersymmetric theories:

$$
V=\frac{1}{2} m^{2} \phi^{2}+\sigma \phi \chi^{2}+h^{2} \phi^{2} \chi^{2}+g^{2} \chi^{4}
$$

where for schematic simplicity the inflaton $\phi$ and the matter scalar $\chi$ are taken to be real scalar fields and the self-coupling of the $\chi$ field is considered to be of moderate strength $10^{-1}<g^{2}<1$. In supersymmetric theories where the decay scalars are standard model chiral scalars which are gauge non-singlets the quartic potential terms in $\chi$ arise as D-terms and the coupling is of gauge coupling strength $g^{2}$. For inflaton-scalar couplings arising from 
superpotentials in supersymmetric theories one has $\sigma=2 \mathrm{hm}$, and the cubic and quartic couplings of the inflaton to $\chi$ are related to each other. In general the superpotential couplings $h$ may be, and in viable supersymmetric inflationary models are usually chosen to be, much smaller than gauge couplings $h \ll g$. The inflaton mass $m$ must be bounded by $m \lesssim 10^{-6}$ in order to be consistent with COBE data on microwave background fluctuations [35].

First let us review the effects of parametric amplification on inflaton decay, ignoring selfinteraction of the decay products. The nonlinear effects that lead to amplified decay act in two different regimes:

[1] $\frac{m^{4}}{\sigma^{2}} \lesssim \phi \lesssim \frac{m^{2}}{\sigma}$ for the cubic coupling and $\frac{m^{4}}{h^{4}} \lesssim \phi^{3} \lesssim \frac{m^{3}}{h^{3}}$ for the quartic coupling. This is the narrow-band resonance case which can be analyzed perturbatively, and the dominant effect is the large occupation number for $\chi$ 's. This case has been considered in $17,8,10$, 11, 18], where it is shown that parametric amplification occurs and there are narrow-band resonances for $\chi$ production at $k=\frac{m}{2}$ and $k=m$ for the cubic and quartic couplings respectively. This is the case for which we will make quantitative estimates of the effect of inclusion of final state self-interaction of the decay products.

[2] $\phi \gtrsim \frac{m^{2}}{\sigma}$ for the cubic coupling and $\phi \gtrsim \frac{m}{h}$ for the quartic coupling. Here perturbation theory is not valid and problem is highly nonlinear. In this case there is broad-band resonance for a large domain of momenta [7, 10] that leads to an explosive decay of the inflaton. As noted above, because of the essentially kinematic nature of the cutoff we expect the finalstate self-interaction effects in this case to be qualitatively similar to those in the narrow band case.

Note that if the cubic and quartic couplings of the inflaton to the decay scalar are of the form arising from a superpotential coupling $[\sigma \simeq 2 h \mathrm{~m}]$, then the conditions for parametric amplification are more general for the cubic coupling and it will dominate the decay. Another important observation is that although most inflatons may decay during a stage of amplified decay this does not lead to the decay of the entire energy density of the inflaton. In the case of narrow-band resonance the decay stops no later than the time when $\phi$ has the minimum value in the abovementioned range $\left(\frac{m^{4}}{\sigma^{2}}\right.$ for the cubic coupling and $\left(\frac{m}{h}\right)^{\frac{4}{3}}$ for the quartic coupling) [7, 凤 , while in the case of broad-band resonance it stops at the time of the transition from broad-band to narrow-band resonance when the decay becomes out of 
equilibrium [7, 10, 25]. After that, the remaining energy density of the inflaton is redshifted as $R^{-3}$ where energy density of relativistic $\chi$ 's is redshifted as $R^{-4}$. The decay of the inflaton will then be completed as in the usual picture, and if the energy density of the inflaton dominates at that time there will be significant dilution of relic densities from the first stage of reheat [25, 16]. In supersymmetric theories this dilution is not, generally, sufficient by itself to solve the problem of overproduction of gravitinos. However, combined with the effect of final state self-interaction, which we consider below, it can successfully resolve the gravitino problem in many models.

Now let us consider generally the changes to the parametric amplification reheat dynamics that arise from the self-interactions of the final state decay products. Since by assumption the inflaton decays to observable sector standard model (s)particles the final state bosons carry gauge quantum numbers. For these fields self-interactions with couplings as strong as the gauge coupling arise at tree-level from D-terms in supersymmetric models 凹,

and at the one-loop level in the non-supersymmetric case. In addition, there are couplings of non-singlet scalars to the gauge fields as well as possible large superpotential couplings. The decay of the inflaton produces quantum fluctuations along the direction of final state particles in field space and drives them to large field values where the effect of self-coupling becomes important. These large field values induce a self-mass for the decay products that subsequently slows down the decay and tends to shut it off .

During the oscillatory regime $\phi \cong \phi_{0} \cos (m t)$, and immediately after the end of inflation $\phi_{0} \sim 10^{-1}$. Subsequently $\phi_{0}$ decreases with time because of decay and Hubble expansion. For $\phi_{0} \lesssim \frac{m}{h}$ the inflaton decays predominantly via the cubic term. As was discussed in [7, 8, 10], in the range $\left(\frac{m}{h}\right)^{2} \lesssim \phi_{0} \lesssim \frac{m}{h}$ parametric amplification occurs and there is a narrow-band resonance for $\chi$ production at $k=\frac{m}{2}$. In this range for the inflaton field we can use the Feynman diagrams for one-particle decay, but the occupation number of final state particles

\footnotetext{
${ }^{1}$ There are, of course, directions in scalar field space which are both D-flat and F-flat in the supersymmetric standard model; in realistic no-scale supergravity models they will in general already have developed Planck scale vevs during inflation [36]. If the decay coupling of the inflaton happened to align along one of these directions then final state interactions of the type we consider would not affect this particular decay mode. More generally the decay products will themselves, in turn, have decay modes along these directions. We consider these issues elsewhere [31].
} 
is non-trivial and must be taken into account in the calcualtions. According to [7, 8, 10] parametric amplification will effectively convert most of the energy density of the inflaton into radiation once it is in the afore-mentioned range. Assuming rapid subsequent thermalization this leads to a reheat temperature $T_{R} \sim m h^{-\frac{1}{2}}$ that is much higher than that of the usual picture $\sim 0.1 m^{\frac{1}{2}} h$ for reasonable values of $h$. The quartic self-coupling of $\chi$ will induce a finite temperature correction to the mass-squared of $\chi$, if they are thermalized, of order $g^{2} T_{R}{ }^{2} \sim g^{2} m^{2} h^{-1}$ at this time which is much larger than $m^{2}$ (as we mentioned above the nonthermal corrections that exist before thermalization are even larger). However at an earlier time $t_{d}$ when the thermal (or non-thermal f) correction is of order $\frac{m^{2}}{4}$ the one-particle decay becomes kinematically forbidden (note that the thermal correction to mass-squared of $\phi$ is of order $h^{2} T_{R}^{2}$ which is normally smaller than $m^{2}$ for $h$ a typical Yukawa type coupling).

The Hubble expansion that subsequently occurs redshifts the correction to the masssquared of $\chi$ as $R^{-2}$ and this causes further decay. As long as $t_{d}<H^{-1}$ these successive steps of expansion, decay and (perhaps) thermalization continue. Eventually the decay is not effective enough to compensate for expansion and there is a delay before the remaining energy density of the inflaton is converted into relativistic particles as in the usual picture, and the decay of the inflaton is completed.

Now let us perform a detailed mode by mode analysis of the effect of quartic self-coupling of final state particles. Consider the potential

$$
\mathrm{V}=\frac{1}{2} m^{2} \phi^{2}+2 h m \phi \chi^{2}
$$

where $\phi$ and $\chi$ are both real scalars. By mode expansion of $\chi$ we derive the following equation for each mode

$$
\ddot{\chi_{k}}+3 \frac{\dot{R}}{R} \dot{\chi_{k}}+\left(k^{2}+4 h m \phi_{0} \cos m t\right) \chi_{k}=0
$$

where a dot denotes differentiation with respect to time, and in this equation for the modes associated with comoving wavenumbers, we are using the physical wavenumber $k$, where

\footnotetext{
${ }^{2}$ Whether the thermal or non-thermal correction should be considered depends on how rapid the thermalization rate is. Details will be discussed shortly.
} 
$R k=k_{\text {comoving }}$ with $R$ the scale factor . For the moment we will ignore the effect of Hubble expansion, since it generally occurs over a longer time scale than that of the effects we consider. We will consider issues of thermalization and Hubble expansion below [for other treatments of the effect of Hubble expansion on the parametric resonance see [7, 8, 11]]. By choosing $z=\frac{m}{2} t$, and in the absence of final state self-interactions we derive a Mathieu equation for the modes of the $\chi$ field

$$
\chi_{k} "+\left(\frac{k^{2}}{\left(\frac{m}{2}\right)^{2}}+\frac{4 h m \phi_{0}}{\left(\frac{m}{2}\right)^{2}} \cos 2 z\right) \chi_{k}=0
$$

where prime denotes differentiation with respect to $z$. In the case of narrow-band resonance (in which we perform our calculations) the Mathieu equation has resonance solutions in the first instability band $\left(\frac{m}{2}\right)^{2}-4 h m \phi_{0}<k^{2}<\left(\frac{m}{2}\right)^{2}+4 h m \phi_{0}$. Modes in this band grow exponentially in time, which one interprets as particle production. The (slow) Hubble expansion will eventually drive the modes out of the instability band, but they spend enough time there to reach a substantial occupation number [18. This time $t_{b}$ can be approximately calculated from $\delta k \sim k H t_{b}$ where $k=\frac{m}{2}$ and $\delta k=8 h \phi_{0}$ is the width of the first instability band which gives $t_{b} \sim \frac{16 h}{m^{2}}$.

The quartic self-coupling $g^{2} \chi^{4}$ will induce an effective mass-squared $m^{2}$ eff for the $\chi$ field and the Mathieu equation with the addition of this self-coupling will be modified to become

$$
\chi_{k} "+\left(\frac{k^{2}+m^{2} e_{\text {ff }}}{\left(\frac{m}{2}\right)^{2}}+\frac{4 h m \phi_{0}}{\left(\frac{m}{2}\right)^{2}} \cos 2 z\right) \chi_{k}=0
$$

In a background of isotropically distributed $\chi$ 's over a narrow band of momenta (which is the case in $\phi \rightarrow \chi \chi$ decay before thermal distribution is achieved) $m_{\text {eff }}^{2} \sim g^{2} \frac{n_{\chi}}{E_{\chi}}$ to the leading order, with $n_{\chi}$ the number density of $\chi$ 's and $E_{\chi}$ their energy [7, 10, 12, 13, 14]. After thermalization, in a thermal background of temperature $T$, we will have the standard result $m_{\text {eff }}^{2} \sim g^{2} T^{2}$.

At $t=0$ when $\phi$ starts oscillating $m^{2}$ eff $=0$ and there is resonance in the band $\left(\frac{m}{2}\right)^{2}-$ $4 h m \phi_{0}<k^{2}<\left(\frac{m}{2}\right)^{2}+4 h m \phi_{0}$. With particle production in this band $m_{\text {eff }}^{2}$ increases and resonance in this band stops when $m^{2}{ }_{\text {eff }}=8 \mathrm{hm} \phi_{0}$ after which the number density of particles produced in this band remains unchanged. There will be, however, resonance in 
the band $\left(\frac{m}{2}\right)^{2}-12 h m \phi_{0}<k^{2}<\left(\frac{m}{2}\right)^{2}-4 h m \phi_{0}$ that also stops when $m_{\text {eff }}^{2}$ increases by a further amount of $8 h m \phi_{0}$. This incremental change of $m^{2}$ eff and smooth transition from one resonance band to the next one continues until $m_{\text {eff }}^{2}=\left(\frac{m}{2}\right)^{2}$, after that there is no resonance solution for physical states, i.e. states with $k^{2}>0$. If we take $E_{\chi} \simeq\left(\frac{m}{2}\right)$ for particles in all bands a change of $8 h m \phi_{0}$ in $m^{2}$ eff correponds to an increase in number density of $\chi^{\prime}$ 's by $\delta n_{\chi} \sim \frac{4 h m^{2} \phi_{0}}{g^{2}}$. For the first band $\left(\frac{m}{2}\right)^{2}-4 h m \phi_{0}<k^{2}<\left(\frac{m}{2}\right)^{2}+4 h m \phi_{0}$ and for $h \phi_{0} \ll m$ the occupation number $f_{\frac{m}{2}}$ required to shift the resonance to the next band is calculated to be

$$
\delta n_{\chi} \sim \frac{1}{(2 \pi)^{3}} f_{\frac{m}{2}} \times 4 \pi\left(\frac{m}{2}\right)^{2} \times 8 h \phi_{0} \sim \frac{4 h \phi_{0} m^{2}}{g^{2}} \Rightarrow f_{\frac{m}{2}} \sim \frac{4 \pi^{2}}{g^{2}}
$$

It has been shown [18] that in the small amplitude limit the analytic result of the decay rate in the $n$-th instability band can also be derived from a physical process, $n$ particles with zero momentum that comprise the classical homogeneous inflaton field annihilating into two final state bosons. In particular, for the first instability band and for $f_{k} \ll 1$ we can use the one-particle decay rate from the standard perturbation theory $\Gamma=\frac{h^{2} m}{8 \pi}$. Narrow-band parametric amplification has been shown to be indeed an induced process [18] which means for $f_{k} \gtrsim 1$ the enhancement of decay rate by the factor $\left(1+f_{k}\right)$ must be taken into account in the particle calculation. The time $t_{1}$ which is needed to reach $f_{\frac{m}{2}}=1$ can be approximately calculated as $\mathrm{\beta}^{3}$

$$
\frac{h^{2} m}{8 \pi} t_{1} m \phi_{0}^{2} \sim \frac{\frac{2 h m^{2} \phi_{0}}{g^{2}}}{\frac{4 \pi^{2}}{g^{2}}} \Rightarrow t_{1} \sim \frac{4}{\pi h \phi_{0}}
$$

and considering the enhancement factor the time $\delta t$ which is needed to reach $f_{\frac{m}{2}}=\frac{4 \pi^{2}}{g^{2}}$ is

$$
\delta t \sim \frac{4}{\pi h \phi_{0}} \ln \frac{4 \pi^{2}}{g^{2}}
$$

This is the time for the resonance in the band $\left(\frac{m}{2}\right)^{2}-4 h m \phi_{0}<k^{2}<\left(\frac{m}{2}\right)^{2}+4 h m \phi_{0}$ to stop. For the next bands $k^{2}$ is smaller and this means that less phase space volume is available for decay products or, equivalently, the occupation number for those bands is

\footnotetext{
${ }^{3}$ Note that $e^{-\Gamma t_{1}} \simeq 1-\Gamma t_{1}$ for $\Gamma t_{1} \ll 1$, which is valid here.
} 
larger. Therefore, more time will be needed for production of $\delta n_{\chi} \sim \frac{4 h m^{2} \phi_{0}}{g^{2}}$ in bands with smaller $k^{2}$, but not greatly so, as the larger occupation numbers are obtained rapidly due the the large coherent final state enhancement. A reasonable lower estimate for the decay time $t_{d}$ to effectively achieve $m^{2}{ }_{\text {eff }} \sim \frac{m^{2}}{4}$ is

$$
t_{d} \sim \frac{\frac{m^{2}}{4}}{8 h m \phi_{0}} \delta t \sim \frac{m}{8 \pi h^{2} \phi_{0}^{2}} \ln \frac{4 \pi^{2}}{g^{2}}
$$

In order to have physically realistic estimates we take $g^{2} \cong 10^{-1}$ in our calculations. This leads to

$$
\delta t \sim \frac{8}{h \phi_{0}} \quad, \quad t_{d} \sim \frac{m}{4 h^{2} \phi_{0}^{2}}
$$

So resonance at each band of width $8 h m \phi_{0}$ ends in a time $\sim \frac{8}{h \phi_{0}}$ and in an approximate time of $\frac{m}{4 h^{2} \phi_{0}{ }^{2}}$ decay effectively stops. We notice that $t_{d}<H^{-1}$ for $\phi_{0} \gtrsim\left(\frac{m}{h}\right)^{2}$.

In this analysis the effect of self-coupling of $\chi$ on the solutions of the Mathieu equation was considered only to the first order, which is reasonable for the case of narrow-band resonance $h \phi_{0} \lesssim m$. The key assumption in our treatment of the Mathieu equation in the presence of the nonlinear term was adiabaticity, i.e. that we can use the instantaneous value of $m^{2}$ eff which is also legitimate since it changes over a time $\delta t \sim \frac{8}{h \phi_{0}}$ which is greater than $m^{-1}$, the period of oscillations of $\phi$, again because we are in the narrow-band resonance regime $h \phi_{0} \lesssim m$.

Our results show major differences from the simple parametric amplification case. The effect of large occupation number for final state particles is not that dramatic here because there is a whole range of resonance bands instead of a single one and the effect of the selfinteraction of the produced particles drives modes out of the resonance bands much faster than the Hubble expansion. Consequently the leading effect that influences the decay is the self-interaction of the decay products, which stops it very early.

So far we have not considered thermalization of decay products and the Hubble expansion. The temperature of the thermal bath after thermalization of $\chi$ 's is calculated from $\mathrm{f}$

\footnotetext{
${ }^{4}$ In general the number density of particles is not the same before and after thermalization but the energy density is conserved.
} 


$$
\frac{\pi^{2}}{30}\left(g_{B}+\frac{7}{8} g_{F}\right) T^{4}=\rho_{\chi}=n_{\chi} E_{\chi} \sim \frac{m^{4}}{16 g^{2}}
$$

where $g_{B}, g_{F}$ are the number of bosonic and fermionic degrees of freedom, respectively. We take the number of degrees of freedom to be the one in the minimal supersymmetric standatd model $\left(g_{B}=g_{F}=74\right)$ which leads to a temperature $T \simeq g^{-\frac{1}{2}} \frac{m}{5}$. For this temperature, however, the correction to mass-squared of $\chi$ is

$$
m_{\text {eff }}^{2} \sim g^{2} T^{2} \sim g \frac{m^{2}}{25}
$$

which is much less than $\left(\frac{m}{2}\right)^{2}$ for $g^{2} \cong 10^{-1}$. This is just what we expected because $m^{2}{ }_{\text {eff }} \sim$ $g^{2} \frac{n_{\chi}}{E_{\chi}}$ will be smaller after thermalization when the number density decreases and the mean energy of particles increases. Therefore if thermalization is effective, it will lower $m^{2}$ eff significantly which leads to further decay and thermalization. This sequence of decay and thermalization stops when $g^{2} T^{2} \sim \frac{m^{2}}{4}$ that is at a temperature $T \sim m$ for $g^{2} \cong 10^{-1}$ if the sequence is completed within a Hubble time.

Considering thermalization of decay products and the Hubble expansion, there are different possibilities depending on the relation among different time scales involved in the problem, $t_{\text {osc }} \sim m^{-1}, \delta t \sim \frac{8}{h \phi_{0}}, t_{d} \sim \frac{m}{4 h^{2} \phi_{0}^{2}}, t_{t h} \sim \frac{32 \pi}{\alpha m}$, 回 and $t_{H} \sim \sqrt{\frac{3}{8 \pi}} \frac{1}{m \phi_{0}}$. The most important thing is that $t_{\text {osc }}$ is considerably smaller than all other time scales as long as we are in the narrow band regime $\phi_{0} \lesssim \frac{m}{h}$. Therefore changes in $m^{2}$ eff caused by decay or thermalization (that can be considered as changes in the background) are adiabatic and our analysis is in principle valid, irrespective of the relation among $t_{d}, t_{t h}, t_{H}$. Regarding these time scales there are different cases:

[1]- $\delta t \lesssim t_{d} \lesssim t_{t h} \lesssim t_{H}$. This occurs when the inequalities $\phi_{0} \gtrsim 5 \times 10^{-3} \frac{m}{h}$ (to have $t_{d} \lesssim t_{t h}$ ), $\phi_{0} \lesssim 3 \times 10^{-5}$ (to have $t_{t h} \lesssim t_{H}$ ) and $\left(\frac{m}{h}\right)^{2} \lesssim \phi_{0} \lesssim \frac{m}{h}$ (to have $t_{d} \lesssim t_{H}$ ) are satisfied which necessarily means $\frac{m}{h} \lesssim 10^{-2}$. In this case the following sequence of events happens: decay of the inflaton to $\chi$ 's, end of the decay, and thermalization of decay products to a temperature $T \sim m$, all in a time scale shorter than the Hubble time. Hubble expansion then

\footnotetext{
${ }^{5} t_{t h}=\Gamma_{t h}{ }^{-1}$ with $\Gamma_{t h} \sim \alpha^{2} \frac{n_{\chi}}{m^{2}}$ is for an out of equilibrium distribution of $\chi$ 's. It is easily seen that for a thermal bath with $T \sim \frac{m}{2 g}$ (which is the highest temperature that can be achieved) $m^{-1} \lesssim t_{t h}$ also.
} 
redshifts $T$ but for $\phi_{0} \gtrsim\left(\frac{m}{h}\right)^{2}$ (assuming $t_{d} \lesssim t_{t h} \lesssim t_{H}$ all through the way) this sequence keeps $T \sim m$. For $\phi_{0}<\left(\frac{m}{h}\right)^{2}$ decay is no longer effective to compensate for expansion and we must wait until a later time when $\phi_{0} \lesssim h^{2}$ and decay is completed as in the standard picture. This second stage of decay dilutes the gravitinos that are produced earlier during the first stage.

[2]- $\delta t \lesssim t_{t h} \lesssim t_{d} \lesssim t_{H}$. This occurs for $\phi_{0} \lesssim \min \left[5 \times 10^{-3} \frac{m}{h}, 3 \times 10^{-5}\right]$ and $\left(\frac{m}{h}\right)^{2} \lesssim \phi_{0} \lesssim \frac{m}{h}$ which is possible only for $\frac{m}{h} \lesssim 5 \times 10^{-3}$. This case is similar to case [1], only because of the faster thermalization the temperature is higher and closer to the maximum possible $\sim m$.

[3]- $\delta t \lesssim t_{d} \lesssim t_{H} \lesssim t_{t h}$. This occurs when $\phi_{0} \gtrsim \operatorname{Max}\left[5 \times 10^{-3} \frac{m}{h}, 3 \times 10^{-5}\right]$ and $\left(\frac{m}{h}\right)^{2} \lesssim \phi_{0} \lesssim \frac{m}{h}$ that is consistent only for $\frac{m}{h} \gtrsim 3 \times 10^{-5}$. In this case decay stops without effective thermalization in a Hubble time, so the distribution of $\chi$ 's is out of equilibrium. Hubble expansion redshifts $m_{\text {eff }}^{2}$ as $R^{-2}$ and further decay compensates for this change. A thermal distribution of particles is not achieved, however, unless $t_{t h} \lesssim t_{H}$ 甲. For $\phi_{0} \lesssim\left(\frac{m}{h}\right)^{2}$ the situation is as in case [1].

[4]- $t_{t h} \lesssim \delta t \lesssim t_{d} \lesssim t_{H}$. This occurs when $\phi_{0} \lesssim 10^{-3} \frac{m}{h}$ and $\left(\frac{m}{h}\right)^{2} \lesssim \phi_{0} \lesssim \frac{m}{h}$ which is possible only for $\frac{m}{h} \gtrsim 10^{-3}$. In this case the decay products thermalize almost instantaneously and there is thermal equilibrium from the very beginning of the decay. Actually both $\delta t$ and $t_{d}$ are much greater than $\sim \frac{15}{h \phi_{0}}$ and $\sim \frac{m}{2 h^{2} \phi_{0}{ }^{2}}$ in this case because rapid thermalization of decay products in the thermal bath keeps the occupation number at each resonance band below one. This means that temperature can be much lower than its maximum $\sim m$ and the situation is very similar to the one in the standard picture of reheating.

Cases [1] and [2] are the most dangerous regarding the problem of gravitino production. In these cases thermal equilibrium can in principle be achieved from the very beginning and can last until the time $H^{-1}=\sqrt{\frac{3}{8 \pi}} m^{-3} h^{2}$. Even in these cases the gravitino overproduction is not that serious since at most we have a thermal bath with temperature $T \sim m$ for a time $t \sim \sqrt{\frac{3}{8 \pi}} m^{-3} h^{2}$, much shorter than $t \sim m^{-2}$ (assuming $m \ll\left(\frac{m}{h}\right)^{2}$ ) which is the case for a radiation-dominated universe with temperature $T \sim m$. Case [3], on the other hand,

\footnotetext{
${ }^{6}$ In this case Hubble expansion moves particles from one band to another one. This slightly lowers the time $\delta t$ spent in those bands because now there is an initial number of particles in each band. This, however, is negligible since $\delta n_{\chi}$ is also redshifted as $R^{-3}$.
} 
is the most secure since the distribution of $\chi$ 's is out of equilibrium, a distribution with less mean energy per particle and higher number density compared with a thermal distribution. Depending on the parameters of the model $m, h$ and strength of the self coupling $g^{2}$ one or all of these cases can happen for $\left(\frac{m}{h}\right)^{2} \lesssim \phi_{0} \lesssim \frac{m}{h}$ but the temperature is at most of order $m$ as long as the inflaton is in this range, and is redshifted after that. Also the thermal and non-thermal corrections to the mass-squared of $\chi$ are always $\lesssim \frac{m^{2}}{4}$.

To compare our results with that of parametric amplification without the effect of final state self-interaction let us consider the case $h=10^{-6}, m=10^{-7}$, where the standard picture predicts $T_{R} \sim 0.1 m^{\frac{1}{2}} h=10^{8} \mathrm{GeV}$. In the simple parametric amplification case almost all the energy density of the inflaton is converted into radiation once $\phi_{0} \lesssim \frac{m}{h}=10^{-1}$ and leads to a very high reheat temperature $T_{R} \sim 10^{15}-10^{16} \mathrm{GeV}$ which, from the point of view of gravitino overproduction, is a disaster. According to our present analysis we are in the case [3] in the above all the way from $\phi_{0}=\frac{m}{h}=10^{-1}$ to $\phi_{0}=\left(\frac{m}{h}\right)^{2}=10^{-2}$. This means that thermalization is not effective for $10^{-2} \lesssim \phi_{0} \lesssim 10^{-1}$ and occurs much later when decay is no longer effective, so the temperature during the first stage of decay is actually lower than $\sim 10^{12} \mathrm{GeV}$. This results in a gravitino number density after the first epoch of decay which is too large by a factor of at most $10^{12}$. The inflaton decay is then completed via a second stage when $\phi_{0} \lesssim \frac{h^{2}}{75} \simeq 10^{-14}$. By this time the temperature of the thermal bath and the momentum of the relativistic particles that might have been produced during the first stage are redshifted by a factor $\left(\frac{10^{-28}}{10^{-4}}\right)^{\frac{1}{3}}=10^{-8}$. The second stage will determine the effective reheat temperature to be $\sim 10^{8} \mathrm{GeV}$, and releases a large amount of entropy that dilutes the gravitinos produced during the first stage of decay by a factor of $\left(\frac{10^{12}}{10^{8}}\right)^{3}=10^{12}$ which is now sufficient as a dilution factor.

As we noted previously, since the effect which we consider is a kinematical cutoff due to the large self-induced plasma mass of the strongly self-interacting final state decay products, we expect to also have similar effects in the broad-band case. Studies of the broad band resonance case [7, 10, 14 indicate efficient production of the decay products only for masses up to about an order of magnitude larger than the inflaton mass, so that when the broad-band resonance has built up a sufficient density of the decay products ( typically in a non-thermal "preheat" distribution) that their self-induced plasma mass exceeds this range, the decay will 
be suppressed. As above the decay will subsequently proceed as thermalization and Hubble expansion reduce the number densities and the induced self-mass for the decay products dips into the accessible range, resulting in a regular, quasi steady-state transfer of energy into the decay products.

Finally, we contrast the effects considered herein with those considered by Khlebnikov and Tkachev [13], who studied the semi-classical non-linear effects of the inflaton decay coupling in a massless $\lambda \phi^{4}$ model. Note that the effects they study have a different and independent origin from those considered here. The effects which we study are specifically due to final state self-interactions of the decay products which are different from, and larger (gauge strength) than, the inflaton decay coupling. If these final-state self-couplings are present, then we saw above that their effects acted to regulate the parametric amplification of inflaton decay.

Returning to the narrow-band case, as treated above, it is useful to ask if there are viable models where we are in the narrow-band regime from the beginning of oscillations, and for which the analysis presented here provides quantitative, as well as qualitative guidance. We note that in a simple chaotic inflation model with potential $V(\phi)=\frac{1}{2} m^{2} \phi^{2}$ inflation ends when $V^{\prime \prime}(\phi) \simeq 24 \pi V(\phi)$ which happens for $\phi \lesssim 10^{-1}$. In the case of primordial supersymmetric inflation or new inflationary models the amplitude of post-inflation oscillations around the global minimium is generically substantially smaller than the Planck scale. Depending on the parameters then, some of these models may satisfy the inequalities necessary to be in the narrow-band resonance regime, or even for the nonlinear effects to be negligible. Interestingly enough, it seems that in some viable supersymmetric models this is indeed the case, and the analysis we have here undertaken is quantitatively valid. We will return to these issues elsewhere [31.

In conclusion, we have seen that the self-interaction of final state bosons of moderate strength, that arises very naturally in supersymmetric models, has an important impact on the decay of observable sector inflatons, besides producing a rapid thermalization rate. As a step towards improved understanding of the reheating process we have considered a simple schematic model representing generic features of supersymmetric theories, with such a final state self-coupling, and have shown that in the case of narrow-band resonance 
the outcome is qualitatively different from that of simple parametric amplification. Here inflaton decay occurs during two stages: one stage that consists of successive steps of partial decay, thermalization and expansion at early times which ends relatively soon, and a second stage as in the standard picture that completes the decay. In the first stage because of the quasi-adiabaticity we were able to show that the temperature and the amplitude of quantum fluctuations of final state particles are at most of the order of the mass of the inflaton (approximately), and the temperature is several orders of magnitude below the naive predictions of parametric amplification. The second stage of decay then determines the final reheat temperature and releases a substantial amount of entropy; is of particular importance in order to dilute the previously produced gravitinos in realistic supersymmetric models.

Note Added

After this paper was submitted a preprint appeared from Prokopec and Roos hep-ph/9610400 who do lattice simulations of inflaton decay. In the case where the decay product field has final state self-interactions of moderate strength their simulations exhibit regulation of the broad-band resonance decay, in agreement with the general analytic arguments we have presented above for the broad-band case. 


\section{Acknowledgements}

We are deeply indebted to both John Ellis and Andrei Linde for enlightening discussions, thoughtful criticism, and ongoing collaboration. This work was supported in part by the Natural Sciences and Engineering Research Council of Canada. In addition, RA wishes to thank the Iranian Ministry of Culture and Higher Education for continuing support.

\section{References}

[1] for a review see: A. D. Linde, "Particle Physics and Inflationary Cosmology", (Harwood, Chur, Switzerland, 1990) and references therein.

[2] A. Dolgov and A.D. Linde, Phys. Lett. 116B, 329 (1982).

L.F. Abbott, E. Farhi, and M. Wise, Phys. Lett. 117B, 29 (1982).

[3] for a review see: L. Kofman, preprint UH-IFA-96-28 and astro-ph/9605155.

[4] A. Dolgov and D. Kirilova, Sov. Nucl. Phys. D51, 273 (1990).

[5] J. Traschen, and R. Brandenberger, Phys. Rev. D42, 2491 (1990).

[6] Y. Shtanov, Ukr. Fiz. Zh. 38, 1425 (1993). (in Russian)

[7] L. Kofman, A. D. Linde, and A. A. Starobinsky, Phys. Rev. Lett. 73, 3195 (1994).

[8] Y. Shtanov, J. Traschen, and R. Brandenberger, Phys. Rev. D51, 5438 (1995).

[9] L. Kofman, A. D. Linde, and A. A. Starobinsky, Phys. Rev. Lett.76, 1011 (1996).

[10] A. D. Linde, private communication (1996).

[11] D. Kaiser, Phys. Rev. D53, 1776 (1996).

[12] I. I. Tkachev, Phys. Lett. 376B, 35 (1996).

[13] S. Yu. Khlebnikov and I. I. Tkachev, Phys. Rev. Lett.77, 219 (1996). 
[14] S. Yu. Khlebnikov and I. I. Tkachev, preprint OSU-TA-20/96 and hep-th/9608458.

[15] A. Riotto and I. I. Tkachev, preprint FERMILAB-Pub-96/88-A and astro-ph/9604444.

[16] E. W. Kolb, A. D. Linde, and A. Riotto, preprint FERMILAB-Pub-96/133-A and hep-ph/9606260.

[17] G. Anderson, A. D. Linde, and A. Riotto, preprint FERMILAB-Pub-96/078-A and hep-ph/9606416.

[18] S. Kasuya and M. Kawasaki, preprint ICRR-Report-360-96-11 and hep-ph/9603317.

[19] D. T. Son, preprint UW-PT-96-01 and hep-ph/9601377.

[20] D. T. Son, preprint UW-PT-96-05 and hep-ph/9604340.

[21] M. Yoshimura, Prog. Theor. Phys. 94, 873 (1995).

[22] M. Yoshimura, preprint TU-96-499, and preprint hep-ph/9603356.

[23] M. Yoshimura, preprint TU-96-500, and preprint hep-ph/9605246.

[24] H. Fujisaki, K. Kumekawa, M. Yamaguchi, and M. Yoshimura, Phys. Rev. D53, 6805 (1996).

[25] H. Fujisaki, K. Kumekawa, M. Yamaguchi, and M. Yoshimura, preprint TU-95-493, and preprint hep-ph/9511381.

[26] D. Boyanovsky, H. J. de Vega, R. Holman, D. -S. Lee, and A. Singh, Phys. Rev. D51. 4419 (1995) .

[27] D. Boyanovsky, M. D'Attanasio, H. J. de Vega, R. Holman, and D. -S. Lee, Phys. Rev. D52. 6805 (1995) .

[28] D. Boyanovsky, M. D'Attanasio, H. J. de Vega, R. Holman, and D. -S. Lee, preprint PITT-95-1755 and hep-ph/9511361. 
[29] D. Boyanovsky, H. J. de Vega, R. Holman, and J.F.J. Salgado, hep-ph/9608205.

[30] D. Boyanovsky, H. J. de Vega, R. Holman, and J.F.J. Salgado, hep-ph/9609007.

[31] R. Allahverdi, B.A. Campbell and J. Ellis, in preparation.

[32] J. Ellis, J. E. Kim, and D. V. Nanopolous, Phys. Lett. 145B, 181 (1984).

[33] M. Kawasaki and T. Moroi, Prog. Theor. Phys. 93, 879 (1995)

[34] for a recent review see: S. Sarkar, preprint OUTP-95-16-P and hep-ph/9602260.

[35] B.A. Campbell, S. Davidson, and K.A. Olive, Nucl. Phys. B399, 111 (1993).

[36] M.K. Gaillard, H. Murayama, and K.A. Olive, Phys. Lett. B355, 71 (1995). 